\title{
Terminal Care for Persons with Advanced Dementia in the Nursing Home and Home Care Settings
}

\author{
SUSAN L. MITCHELL, M.D., M.P.H., FRCPC, ${ }^{1,2}$ JOHN N. MORRIS, Ph.D. ${ }^{1}$ \\ PIL S. PARK, Ph.D., ${ }^{3}$ and BRANT E. FRIES, Ph.D ${ }^{3-5}$
}

\begin{abstract}
Background: Many older persons with advanced dementia receive terminal care in nursing homes, others remain in the community with home care services.

Objectives: To describe and compare the end-of-life experience of persons dying with advanced dementia in the nursing home and home care settings.

Design: Retrospective cohort study.

Setting/Subjects: Persons 65 years or older with advanced dementia who died within 1 year of admission to either a nursing home in Michigan between July 1, 1998 until December 31, 2000 ( $n=2730)$, or the state's publicly funded home and community-based services from October 1, 1998 until December 31, $2001(n=290)$.

Measurements: Data were derived from the Minimum Data Set (MDS)-Nursing home Version 2.0 for the institutionalized sample, and the MDS-Home Care for the community-based sample. Variables from the MDS assessment completed within 180 days of death were used to describe the end-of-life experiences of these two groups.

Results: Nursing home residents dying with advanced dementia were older, had greater functional impairment, and more behavior problems compared to home care clients. Few subjects in the nursing home $(10.3 \%)$ and home care $(15.6 \%)$ cohorts were perceived to have less than 6 months to live. Only $5.7 \%$ of nursing home residents and $10.7 \%$ home care clients were referred to hospice. Hospitalizations were frequent: nursing home, 43.7\%; home care, 31.5\%. Pain and shortness of breath were common in both settings. End-of-life variables independently associated with nursing home versus home care included: hospice (adjusted odds ratio [AOR] 0.26, 95\% confidence interval [CI], 0.16-0.43), life expectancy less than 6 months (AOR 0.31; 95\% CI, 0.20-0.48), advance directives (AOR, 1.48; 95\% CI, 1.11-1.96), pain (AOR, 0.38 ; 95\% CI, 0.29-0.50), shortness of breath (AOR 0.20; 95\% CI (0.13-0.28), and oxygen therapy (AOR, 2.47; 95\% CI, 1.51-4.05).

Conclusions: Persons dying with advanced dementia admitted to nursing homes have different characteristics compared to those admitted to home care services. Their end-of-life experiences also differ in these two sites of care. However, palliative care was not optimal in either setting.
\end{abstract}

\footnotetext{
${ }^{1}$ Hebrew Rehabilitation Center for Aged Research and Training Institute, Boston, Massachusetts.

${ }^{2}$ The Department of Medicine of Beth Israel Deaconess Medical Center, and Division on Aging, Harvard Medical School, Boston, Massachusetts.

${ }^{3}$ Institute of Gerontology and ${ }^{4}$ School of Public Health, University of Michigan, ${ }^{5}$ Ann Arbor VA Medical Center, Ann Arbor, Michigan.
} 


\section{INTRODUCTION}

B Y THE YEAR 2030, more than 13 million Americans will suffer with dementia, a progressive and incurable condition. ${ }^{1}$ Recent work has shown that the site of death has important implications for the quality of terminal care. ${ }^{2}$ Little is known about the end-of-life experience of persons dying with advanced dementia in different care settings.

The majority of individuals with advanced dementia receive their end-of-life care in nursing homes. ${ }^{3-6}$ However, a substantial proportion of terminal care in dementia is provided in the community with home health services. ${ }^{3-6}$ Many older persons prefer to stay at home as long as possible near the end-of-life. ${ }^{7,8}$ Patients' preferences for the site of terminal care shift toward institutionalization when they feel that they can no longer receive adequate palliation in their homes and they are too burdensome for their families. ${ }^{8}$ The unique care requirements in end-stage dementia can be particularly challenging for informal caregivers. ${ }^{6}$

To promote informed decision-making regarding the site of terminal care, the experience of persons dying with advanced dementia across various settings needs to be better described. Therefore, the objective of this study was to examine and contrast the end-of-life experience of severely demented individuals who died within one year of admission to either a nursing home or a community-based home care service. In order to achieve this objective, we conducted a retrospective study using data from the Minimum Data Set (MDS) ${ }^{9-13}$ The MDS is a standardized, comprehensive, assessment instrument that collects data on a broad range issues pertinent to end-of-life care including: burdensome symptoms, advance directives, health service utilization, and treatments. In Michigan, the MDS is mandated for use in all nursing homes ${ }^{9-12}$ as well as by the state's publicly funded home care services, ${ }^{13}$ providing a unique opportunity to describe terminal care in these two settings.

\section{METHODS}

\section{Data sources}

The study was exempt from review by the Harvard Medical School Office for Subject Protection.
Two data sources from the state of Michigan were used to identify and describe the study population: (1) The Minimum Data Set-Nursing Home (MDS-NH) Version 2.0 for the institutionalized sample $^{9-12}$ and (2) the MDS-Home Care (MDSHC) for the community-based sample. ${ }^{13}$

The MDS is mandated for use in all nursing homes (MDS-NH) nationwide. ${ }^{9-12}$ As well, Michigan has mandated the use of a compatible assessment system, the MDS-HC, ${ }^{13}$ for use in its Michigan Choice Waiver for the Elderly and Disabled program. The waiver program provides a wide range of home care agency-based services to persons who would otherwise be eligible for nursing home care. The definitions and coding conventions for the variables used in this study were identical in the MDS-NH and MDS-HC instruments, unless otherwise noted. Data were collected by trained nurses in both settings. Interrater reliabilities for all the MDS items are adequate for research purposes. ${ }^{9-12}$ In the nursing home, full MDS-NH assessments are completed at the time of admission, annually, and whenever there is a significant change in health status. An abbreviated version of the MDS is completed on a quarterly basis for clinically stable patients. In the home care program, full MDS-HC assessments are performed quarterly.

In this study, MDS-NH data were collected between July 1, 1998 and December 31, 2000 from nursing home residents living in all facilities in Michigan ( $n=121,129$ residents, $n=458$ facilities). The MDS-HC data were collected between October 1, 1998 and December 31, 2001 from all persons enrolled in the state's waiver home care management programs ( $n=23,095$ individuals).

\section{Population}

Persons aged 65 years or older with advanced dementia who died within 12 months of admission to either a nursing home or home care program were identified. Only patients who had a full MDS assessment completed within 180 days of death were included. Advanced dementia was defined as having a diagnosis of dementia (Alzheimer's disease or other causes) and a Cognitive Performance Score (CPS) of 5 or 6 on the MDS assessment completed at the time of admission to the nursing home or home care program. ${ }^{11}$ The Alzheimer's disease and dementia diagnoses have been used for epidemiological research, 15 and have intraclass coefficients of 0.89 and 0.79 , 
respectively. ${ }^{16}$ The CPS is a validated measure of cognitive impairment that uses five MDS variables to group individuals into seven hierarchical cognitive performance categories (0-6) $\cdot{ }^{14} \mathrm{~A}$ CPS of 5 or 6 corresponds to a Mini Mental Status Examination score of 5 or less.

We wanted to identify older persons with advanced dementia who died within 12 months of admission to either a nursing home or home care program. Therefore, subjects who had MDS information available 12 months after their admission were excluded. For the remaining subjects, death information was obtained from the Michigan Death Registry. The following variables were used to merge the death information with the MDS-NH and MDS-HC databases: first name, middle name, last name, gender, birth date, race, and social security number. If a complete match was found and the death date was within 12 months of the subjects' admission date, they were included in the study. If a complete match was not found or if the subjects' death date was beyond 12 months of their admission date, then they were excluded.

\section{Definition of variables}

Demographic data, functional status, and the presence of other common terminal conditions were derived from the MDS assessments completed at the time of admission to either the nursing home or home care program. Demographic data included the following: age, days between the admission and death date, race/ethnicity (white versus other), and gender. Functional status was quantified using the MDS Activity of Daily Living (ADL) Long Form Scale (0-28). ${ }^{17}$ The scale measures functional ability in seven domains. A score of 28 represents complete dependence in all seven domains. The presence of behavior problems (wandering or physical agitation) on admission was also determined. Patients with diagnoses of cancer (MDS does not distinguish the type of cancer), congestive heart failure and chronic obstructive pulmonary disease were identified.

In order to describe the dying experience in advanced dementia, we selected outcome variables from the MDS thought, a priori, to represent important features of end-of-life care based on our knowledge of the literature $(2-5,18)$ and clinical experience. These data were derived from the last MDS assessment completed prior to death, unless otherwise noted. Variables were categorized as follows: health services utilization, advance care planning, nonpalliative interventions, signs and symptoms, and other treatments. The number of days between the date of the last MDS assessment and death was also determined.

In the category of health services utilization, we examined whether or not subjects experienced a hospitalization or emergency room visit within the 90 days prior to their last MDS assessment. Subjects referred to hospice at any time while being cared for at the nursing home or at home were identified.

In the area of advance care planning, we examined whether or not the subjects had an advance directive. The MDS-HC does not include data on specific advance directives. Therefore, home care subjects were considered to have an advance directive if the response to the following MDS-HC item was positive: "Does the patient have an advance directive (e.g., request for donot-hospitalize)." Nursing home residents were considered to have an advance directive if they had either a request not to be resuscitated (DNR) or hospitalized (DNH) recorded on the MDS-NH.

Nonpalliative interventions included the use of feeding tubes and intravenous therapy (fluids or medications). Other treatments included oxygen therapy (intermittent or continuous) during the 14 days prior to the last assessment and indwelling bladder catheters. We also examined whether or not subjects received any of the following medications in the 7 days prior to their last assessment: antipsychotics, antianxiety agents and antidepressants. Signs and symptoms included pneumonia, pain (daily or almost daily), shortness of breath, and pressure ulcers (with at least some loss of skin integrity). Finally, the presence of chewing or swallowing difficulties were determined.

\section{Statistical analysis}

All analyses were performed using SAS Version 8.1 for Windows (19). In order to compare the end-of-life experience in advanced dementia in the nursing home and at home, care setting was the main independent variable in all analyses. Bivariate analyses were conducted comparing subject characteristics and end-of-life outcomes in these two settings. $\chi^{2}$-Tests and two-sample $t$ tests were used to analyze dichotomous and continuous variables, respectively. ADL score was categorized into three groups based on terciles; 0-20 (referent), 21-27, and 28. 
End-of-life outcomes that differed significantly $(p<0.05)$ between the nursing home and home care settings in the bivariate analyses, were selected for multivariate analysis using logistic regression. Age, race, gender, functional status, ethnicity, cognitive status (CPS score of 6 versus 5), and days between admission and death were included as covariates in all multivariate models. Other potential confounders were included as covariates in individual models depending on the outcome being examined. Variables were only considered as potential confounders if the bivariate association between the location of care and the individual variable differed by a $p$ value $\leq 0.10$. Diagnoses or conditions included as covariates in particular models included (dependent variables indicated in parentheses): pneumonia and chronic obstructive pulmonary disease (hospitalization and shortness of breath models), pain and shortness of breath (hospice referral), chewing or swallowing problems (tube feeding), shortness of breath (oxygen therapy, chronic obstructive lung disease), and behavior problems (antianxiety medication).

\section{RESULTS}

\section{Subject characteristics}

There were 3483 nursing home residents and 314 home care clients with advanced dementia who died within 1 year of admission. Among these subjects, we included the 2730 (78\%) nursing home residents and 290 (92\%) of home care clients who had full MDS assessments 180 days prior to death. The nursing home residents excluded from the study because they did not have a full MDS assessment completed within 180 days of death $(n=753,12 \%)$ did not differ significantly from those included in the study with respect to the following baseline characteristics: age, gender, ADL score, CPS, days between admission and death, and other potentially terminal diagnoses (i.e., cancer, chronic obstructive pulmonary disease, and congestive heart failure). No subjects were included in both the nursing home and home care cohorts. The mean number of days between the last MDS assessment prior to death and death did not differ significantly between the nursing home and home care cohorts (51.0 \pm [SD] 50.0 versus $54.7 \pm$ [SD] 35.5 days, $p=0.11$ ).

At the time of admission, nursing home residents with advanced dementia were more likely to have the following characteristics compared to home care clients: older age, fewer days between admission and death, total functional dependence, white race (versus non-white), a CPS score of 6 (versus 5), and behavior problems. Gender did not differ between the two groups (Table 1).

\section{Health services utilization}

The frequencies and bivariate analyses comparing the end-of-life characteristics of older per-

Table 1. Subject Characteristics

\begin{tabular}{lccc}
\hline Characteristic & Home care $(\mathrm{n}=290)$ & Nursing home $(\mathrm{n}=2730)$ & $\mathrm{p}$ value \\
\hline Age on admission (mean years \pm SD) & $81.7 \pm 7.4$ & $83.4 \pm 7.1$ & 0.01 \\
Female (\%) & 65.0 & 63.0 & 0.32 \\
White (\%) & 72.9 & 82.8 & $<0.001$ \\
ADL score (level of dependence) & & 24.3 & $<0.001$ \\
$\quad 0-20$ & 70.2 & 36.2 & $<0.001$ \\
$21-27$ & 23.1 & 39.3 & $<0.001$ \\
28 (total dependence) & 6.7 & $173.7 \pm 286.4$ & $<0.001$ \\
Days between death and admission (mean \pm SD) & $306.24 \pm 301.7$ & 57.1 & $<0.001$ \\
Cognitive Performance Score $=6$ (versus 5) (\%) & 34.6 & 31.1 & $<0.001$ \\
Behavior problems (\%) & 10.6 & 11.4 & 0.23 \\
Other diagnoses & & 15.6 & 0.07 \\
$\quad$ Cancer (\%) & 13.7 & 29.6 & 0.94 \\
Chronic obstructive pulmonary disease (\%) & 11.6 & 29.6 & \\
Congestive heart failure (\%) & & & \\
\hline
\end{tabular}

aADL, activities of daily living. Total ADL score is the sum of scores in each of 7 domains of function including bed mobility, dressing, toileting, transfer, eating, grooming, and locomotion. Each is scored on a 5 point scale $(0$, independent; 1, supervision; 2, limited assistance; 3, extensive assistance; and 4, total dependence).

${ }^{b}$ Cognitive Performance Score of 6 is defined as very severe cognitive impairment, a score of 5 is defined as severe cognitive impairment.

SD, standard deviation. 
sons with advanced dementia in the nursing home and at home are presented in Table 2. Table 3 shows the results of the multivariate analyses.

Hospital admissions just prior to death were common in both groups, but more frequent among the nursing home cohort $(43.7 \%$ versus $31.5 \%, p<0.001$ ), Hospitalization rates did not differ between the two groups after multivariate adjustment. Few subjects dying with advanced dementia were referred to hospice. Hospice referral was significantly lower among nursing home residents compared to home care clients $(5.7 \%$ versus $13.1 \%, p<0.001)$, even after adjusting for potential confounders. Emergency room visits did not differ between the two groups.

\section{Advance care planning}

A higher proportion of nursing home residents had advance directives compared to subjects receiving home care $(57.4 \%$ versus $39.4 \%, p<$ 0.001). Within 180 days of death, few subjects in either cohort were perceived by their caregivers as having life expectancy of less than 6 months, however the proportion of subjects with this poor prognosis was significantly lower among nursing home residents $(10.3 \%$ versus $15.6 \%, p<0.02)$.
These associations remained significant after multivariate adjustment.

\section{Nonpalliative interventions}

Among nursing home residents with advanced dementia, $27.2 \%$ died with a feeding tube, compared to $11.9 \%$ of subjects in the home care program $(p<0.001)$. However, the majority of tubefed subjects had feeding tubes at the time of admission to the nursing home $(83 \%)$ and home care programs $(78 \%)$. Few subjects in the nursing home $(3.6 \%)$ and home care cohorts $(2.8 \%)$ were reported to receive intravenous therapy just prior to the death $(p=0.52)$

\section{Signs and symptoms}

It was common for older persons with advanced dementia to suffer with distressing signs and symptoms near the end-of-life in both care settings. However, compared to home care clients, subjects in the nursing home were reported to have less pain ( $37.1 \%$ versus $53.4 \%, p<$ $0.001)$ and shortness of breath $(12.7 \%$ versus $29.7 \%, p<0.001)$, even after multivariate adjustment: pain (adjusted odds ratio [AOR], 0.38; 95\%

Table 2. Bivariate Analysis

\begin{tabular}{|c|c|c|c|}
\hline Characteristic (\%) & Home care $(\mathrm{n}=290)$ & Nursing home $(\mathrm{n}=2730)$ & $\mathrm{p}$ value \\
\hline \multicolumn{4}{|l|}{ Health services utilization } \\
\hline Hospitalization within the previous 90 days & 31.5 & 43.7 & $<0.001$ \\
\hline Emergency department visit within the previous 90 days & 13.1 & 11.4 & 0.41 \\
\hline Hospice referral (any time prior death) & 13.1 & 5.7 & $<0.001$ \\
\hline \multicolumn{4}{|l|}{ Advance care planning } \\
\hline Advance directive & 39.4 & 57.4 & $<0.001$ \\
\hline Perceived life expectancy $<6$ months & 15.6 & 10.3 & 0.02 \\
\hline \multicolumn{4}{|l|}{ Nonpalliative interventions } \\
\hline Feeding tube & 11.9 & 27.2 & $<0.001$ \\
\hline Intravenous therapy & 2.8 & 3.6 & 0.52 \\
\hline \multicolumn{4}{|l|}{ Signs and symptoms } \\
\hline Pneumonia & 5.2 & 16.9 & $<0.001$ \\
\hline Daily or almost daily pain & 53.4 & 37.1 & $<0.001$ \\
\hline Shortness of breath & 29.7 & 12.7 & $<0.001$ \\
\hline Pressure ulcers & 19.7 & 35.0 & $<0.001$ \\
\hline Depression & 22.3 & 20.5 & 0.49 \\
\hline \multicolumn{4}{|l|}{ Other treatments } \\
\hline Oxygen therapy & 12.5 & 24.4 & $<0.001$ \\
\hline Foley catheter & 15.8 & 29.4 & $<0.001$ \\
\hline Antipsychotic medications & 19.7 & 22.7 & 0.35 \\
\hline Antianxiety medications & 20.7 & 15.9 & 0.05 \\
\hline Antidepressant medications & 24.7 & 21.5 & 0.21 \\
\hline
\end{tabular}

Characteristics on the last Minimum Data Set assessment prior to death of older persons with advanced dementia cared for in a nursing home $(n=2730)$ versus at home $(n=290)$. 
Table 3. Multivariate Analysis

\begin{tabular}{ll}
\hline Characteristic & Adjusted $O R^{\mathrm{a}}$ \\
\hline $\begin{array}{l}\text { Health services utilization } \\
\text { Hospitalization in the } \\
\text { previous } 90 \text { days }\end{array}$ & $1.30(0.98,1.74)^{\mathrm{b}}$ \\
$\quad \begin{array}{l}\text { Hospice referral (any } \\
\quad \text { time prior to death) }\end{array}$ & $0.26(0.16,0.43)^{\mathrm{c}}$ \\
Advance care planning & \\
$\quad$ Advance directive & $1.48(1.11,1.96)$ \\
$\quad$ Perceived life expectancy & $0.31(0.20,0.48)$ \\
$\quad<6$ months & \\
Nonpalliative interventions & $1.70(1.01,2.84)^{\mathrm{d}}$ \\
$\quad$ Feeding tube & \\
Signs and symptoms & $0.38(0.29,0.50)$ \\
$\quad$ Daily or almost daily pain & $0.20(0.13,0.28)^{\mathrm{b}}$ \\
$\quad$ Shortness of breath & $2.86(1.62,5.04)$ \\
$\quad$ Pneumonia & $1.46(1.04,2.03)$ \\
$\quad$ Pressure ulcers & \\
Other treatments & $2.47(1.51,4.05)^{\mathrm{e}}$ \\
$\quad$ Oxygen therapy & $1.06(0.73,1.53)$ \\
$\quad$ Foley catheter & $0.63(0.44,0.89)^{\mathrm{f}}$ \\
$\quad$ Antianxiety medications &
\end{tabular}

aAll odds ratios are adjusted for age, race (white versus non-white), activities of daily living score on admission, days between admission and death, and Cognitive Performance Score (6 versus 5).

${ }^{\mathrm{b}}$ Hospitalization and shortness of breath models also adjusted for presence of pneumonia and chronic obstructive pulmonary disease.

'Hospice models also adjusted for pain and shortness of breath.

dFeeding tube model also adjusted for the presence of chewing and swallowing problems.

eOxygen therapy model also adjusted for the presence of shortness of breath and chronic obstructive pulmonary disease.

${ }^{\mathrm{f}}$ Antianxiety medication model also adjusted for presence of behavior problems.

Characteristics on the last Minimum Data Set assessment prior to death of older persons with advanced dementia cared for in a nursing home $(n=2730)$ versus at home $(n=290)$.

confidence interval [CI], 0.29-0.50); shortness of breath (AOR, $0.20 ; 95 \% \mathrm{CI}, 0.13-0.28$ ). In order to explore the possibility that the nursing home cohort had less pain due to greater functional disability (i.e., reduced mobility resulting in less opportunity to elicit pain), a stratified analysis was conducted. Pain was significantly more likely in the home care cohort in all three strata of ADL score.

Many persons with advanced dementia died with pressure ulcers in both the nursing home $(35.0 \%)$ and home care $(19.7 \%)$ settings. Pressure ulcers were more likely among the nursing home residents after adjusting for potential con- founders (AOR, 1.46; 95\% CI, 1.04-2.03). Pneumonia was also significantly more likely among the nursing home residents. Depression was common in both care settings, affecting approximately one fifth of all subjects. The likelihood of depression did not differ between the two groups.

\section{Other treatments}

Subjects were more likely to receive oxygen therapy if they resided in the nursing home $(24.4 \%$ versus $12.5 \%, p<0.001)$, even after adjusting for the presence of shortness of breath (AOR, 2.47; 95\% CI, 1.51-4.05). Although shortness of breath was more common in the home care cohort, this group received less oxygen therapy. In addition, nursing home residents were less likely to receive antianxiety medications compared to the home care clients, even after adjusting for behavior problems. Other treatments, including the use of antipsychotics medications, antidepressants and Foley catheters, did not significantly differ between the two groups.

\section{DISCUSSION}

This study reports the end-of-life experience of older persons with advanced dementia who died within 1 year of admission to a nursing home or home care program. Several findings are notable. First, the characteristics of persons admitted to these two care settings differ such that severely demented persons admitted to nursing homes have more functional disability, behavior problems, and feeding tubes compared to those who remain at home. Second, the palliative care provided to this population is not optimal in either setting: most patients are not recognized as dying, hospice referrals are infrequent, and hospitalizations, burdensome treatments, and potentially treatable distressing symptoms are common just prior to death. Finally, several palliative care outcomes in advanced dementia may be more favorable in the nursing home. Reported pain and shortness of breath were less common in the nursing home subjects compared to home care cohort, whereas advance directives and the use of oxygen therapy were more common in the nursing home setting. 
Few previous studies have described the endof-life experience of older persons with advanced dementia in various care settings. ${ }^{3-6,18,20,21}$ Our study corroborates earlier observations that persons with advanced dementia admitted to nursing homes have a greater skilled nursing needs compared to those who are managed at home. In particular, behavior problems and functional disability are consistently associated with institutionalization at all stages of dementia. 5,22

Our study further supports growing expert opinion and empiric data suggesting that palliative care for advanced dementia is sub-optimal across all care settings including nursing homes, ${ }^{4,5,18}$ hospitals, ${ }^{20,21}$ assisted living/residential facilities, ${ }^{4}$ and home. ${ }^{3,5,6}$ Advanced dementia is often not recognized as a terminal illness. ${ }^{18,20,21}$ Moreover, it is uncommon for persons dying with end-stage dementia to be referred to hospice. ${ }^{5,23-25}$ The smaller proportion of hospice referrals in the nursing home may further reflect practical barriers related to contracting hospice services in nursing facilities, or the perception by nursing home staff that additional palliative care expertise is not needed. Hospice services in nursing homes have been shown to improve pain management, promote advance care planning, and reduce the use of invasive treatments. ${ }^{26,27}$ Moreover, older persons receiving home hospice have been shown to have a better dying experience compared to those receiving terminal care in a nursing home or at home with nursing services. ${ }^{2}$

Many family members and health care providers feel palliation should be the primary goal of care for persons with advanced dementia. ${ }^{28}$ We have shown, as have others, that potentially treatable distressing symptoms are common among persons dying with advanced dementia. ${ }^{4-6,18}$ Our finding that $53.4 \%$ of home care clients had daily or almost daily pain, is similar to the proportion reported by family caregivers of persons dying with dementia $(62.6 \%){ }^{6}$ Pain is often underreported in advanced dementia when assessed with standard pain scales and MDS items. ${ }^{29,30}$ Therefore, the number of subjects suffering with pain may even be higher than reported. It is notable that nursing home residents were more likely to receive oxygen therapy and had less reported shortness of breath compared to the home care cohort. Oxygen therapy would be considered reasonable treatment to manage terminal dyspnea associated with hypoxemia. As such, our finding supports earlier work suggesting that palliative treatments may be more readily available in an institutionalized setting. ${ }^{4}$ The avoidance of burdensome hospital admissions is another theoretical advantage of the nursing homes' ability to provide the 24-hour care. We found, as have others ${ }^{3-6,20,21}$ that hospitalizations are very common for persons with advanced dementia, regardless of care setting.

This study has some limitations that deserve comment. First, our data describe the patients' experience in the primary setting where they were cared for near the end-of-life, but not necessarily at the location of death. Second, there may be some inaccuracies in the recording MDS data. The potential for misclassification is minimized by uniform data collection by trained staff and explicitly defined variables in a coding manual. While most misclassification is likely to be nondifferential between the home care and nursing home settings, there may be some reporting bias of symptoms in our two cohorts. For example, nursing home staff may be more reluctant to report pain, one of several MDS quality indicators used to rate nursing homes nationwide. Third, although we examined a well-defined cohort with advanced dementia and used multivariate techniques, we may not have adjusted for all the clinical differences between the home care and nursing home cohorts to explain our findings. Fourth, many important end-of-life outcomes are not captured in the MDS, including family satisfaction with end-of-life care and the process of shared decision-making. Fifth, $12 \%$ of the nursing home residents who died within a year of admission were excluded because a full MDS assessment was not completed within 180 days of death. Although these residents were similar to those who met inclusion criteria at the time of admission, it is possible that these groups differed with respect to their end-of-life experience. Finally, our data are limited to the state of Michigan and may not be generalizable to other states where home care services and nursing home care differ.

Many factors influence the location of terminal care for persons with advanced dementia including patient preferences and care needs, availability of formal and informal services, financial resources, and caregiver burden. As patients, families and practitioners weigh these varying influences, it behooves the health care community to improve the care of those dying with this condi- 
tion in whichever care setting they ultimately spend their final days. This study underscores the need to promote better palliative care in advanced dementia in the home and nursing home settings.

\section{ACKNOWLEDGMENTS}

Supported by the Hebrew Rehabilitation Center for Aged (HRCA) Research and Training Institute, the Marcus Applebaum Fund at the HRCA, the Harvard Older American Independence Center Grant (AG08812) from the National Institute on Aging, Bethesda, MD and InterRAI. Dr. Mitchell is supported by the NIH-NIA Mentored Patient Research Award (K23AG20054).

\section{REFERENCES}

1. Hebert LE, Scherr PA, Bienias JL, Bennett DA, Evans DA: Alzheimer's Disease in the US population: Prevalence estimates using the 2000 Census. Arch Neurol 2003;60:1119-1122.

2. Teno JM, Clarridge BR, Casey V, Welch LC, Wetle T, Shield R, Mor V: Family perspectives on end-of-life care at the last place of care. IAMA 2004;291:88-93.

3. Collins, Ogle K: Patterns of predeath service use by dementia patients with a family caregiver. J Am Geriatr Soc 1994;42:719-722.

4. Sloane PD, Zimmerman S, Hanson L, Mitchell CM, Riedel-Leo C, Cutis-Buie V: End-of-life care in assisted living and related residential care settings: comparison with nursing homes. I Am Geriatr Soc 2003; 51:1587-1594.

5. Volicer L, Hurley AC, Blasi ZV: Characteristics of dementia end-of-life care across settings. Am J Hosp Palliat Care 2003;20:191-200.

6. Schulz R, Mendelsohn AB, Haley WE, Mahoney D, Allen RS, Zhang S, Thompson L, Belle SH: End-of-life care and the effects of bereavement on family caregivers of persons with dementia. N Engl J Med 2003; 349:1936-1942.

7. Pritchard RS, Fisher ES, Teno JM, Sharp SM, Reding DJ, Knaus WA, Winnberg JE, Lynn J: Influence of patient preferences and local health system characteristics on the place of death. I Am Geriatr Soc 1998; 46:1242-1250.

8. Fried TR, Doorn van C, O'Leary JR, Tinetti ME, Drickamer MA: Older persons' preferences for site of terminal care. Ann Intern Med 1999;131:109-112.

9. Hartmaier SL, Sloane PD, Guess HA, Koch GG, Mitchell CM, Phillips CD: Validation of the Minimum Data Set Cognitive Performance Scale: Agreement with Mini-Mental State Examination. J Gerontol A Biol Sci Med Sci 1995;50:M128-133.
10. Morris JN, Hawes C, Fries BE, Phillips CD, Mor V, Katz S, Murphy K, Drugovich ML, Friedlob AS: Designing the National Assessment Instrument for nursing homes. Gerontologist 1990;30:293-307.

11. Morris JN, Nonemaker S, Murphy K, Hawes C, Fries BE, Phillips C: A commitment to chance: Revision of HFCA's RAI. J Am Geriatr Soc 1997;45:1011-1016.

12. Mor V, Angelelli J, Jones R, Roy J, Moore T, Morris JN: Inter-rater reliability of nursing home quality indicators in the U.S. BMC Health Services Research 2003;3:20-33, 〈www.biomedcentral.com/1472-6963/ $3 / 20\rangle$

13. Landi, F, Tua E, Onder G, Carrara B, Sgadari A, Rinaldi C, Gambassi G, LaHanzio F, Bernabei R, Silvernet-HC Study Group of Bergamo: Minimum data set for home care: A valid instrument to assess frail older people living in the community. Med Care 2000; 38:1184-1190.

14. Morris JN, Fries BE, Mehr DR, Hawes C, Phillips C, Mor V, Lipsitz LA: MDS Cognitive Performance Scale. J Gerontol. 1994;49:M174-82.

15. Lapane KL, Gambassi G, Landi F, Sgadari A, Mor V, Bernabei R. Gender differences in predictors of mortality in nursing home residents with Alzheimer's disease. Neurology 2001;56:650-654.

16. Hawes C, Morris JN, Phillips CD, Mor V, Fries BE, Nonemaker S. Reliability estimates for the Minimum DataSet for nursing home resident assessment and care screening (MDS). Gerontologist 1995;35:172-178.

17. Morris JN, Fries BE, Morris SA. Scaling ADLs with the MDS. J Gerontol 1999;54:M546-53.

18. Mitchell SL, Kiely DK, Hamel MB. Dying with advanced dementia in the nursing home. Arch Intern Med. 2004:164:321-326

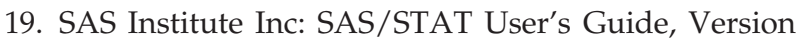
8.1. The UNIVARIATE, FREQUENCY, and LOGIST procedures. Vol. 1 and 2. Cary, NC: SAS Institute Inc.; 2000.

20. Ahronheim JC, Morrison RS, Baskin SA, Morris J, Meier DE: Treatment of the dying in the acute care hospital: Advanced dementia and metastatic cancer. Arch Intern Med 1996;156:2094-2100.

21. Morrison RS, Siu AL: Survival in end-stage dementia following acute illness. JAMA 2000;284:47-52.

22. Yaffe K, Fox P, Newcomer R, Sands L, Lindquist K, Dane K, Covinsky KE: Patient and caregiver characteristics and nursing home placement in patients with dementia. JAMA 2002;287:209-207.

23. Casarett DJ, Hirschman KB, Henry MR. Does hospice have a role in nursing home care as the end-of-life? I Am Geriatr Soc 2001;49:1493-1498

24. Hanrahan P, Luchins DJ: Access to hospice programs in end-stage dementia: a national survey of hospice programs. J Am Geriatr Soc 1995;43:56.

25. Christakis NA, Escarce JJ: Survival of Medicare patients after enrollment in hospice programs. N Engl J Med 1996:335;172-178.

26. Miller SC, Mor V, Wu N, Gozalo P, Lapane K: Does receipt of hospice care in nursing homes improve the 
management of pain at the end of life? J Am Geriatr Soc 2002;50:507-515.

27. Miller SC, Gozalo P, Mor V. Outcomes and utilization for hospice and non-hospice nursing facility decedents. U.S. Department of Health and Human Services (HHS), Office of Disability and Long-Term Care Policy (DALTCP) and the Urban Institute research studies. 2000: Washington, D.C. (aspe.hhs.gov/daltcp/ home.htm $\rangle$ (Last accessed October 2, 2004).

28. Luchins DJ, Hanrahan P: What is appropriate health care for end-stage dementia? J Am Geriatr Soc 1993; 41:25-30.

29. Ferrell BA, Ferrell BR, Rivera L: Pain in cognitively impaired nursing home patients. I Pain Symptom Manage 1995;10:591-598.
30. Fries BE, Simon SE, Morris JN, Flodstrom C, Bookstein FL: Pain in US nursing homes: Validating a pain scale for the minimum data set. Gerontologist 2001; 41:173-179.

Address reprint requests to: Susan L. Mitchell, M.D., M.P.H., FRCPC Hebrew Rehabilitation Center for Aged 1200 Centre Street Boston, MA 02131

E-mail: smithchell@mail.hrca.harvard.edu 


\section{This article has been cited by:}

1. Deborah Birch, Jan Draper. 2008. A critical literature review exploring the challenges of delivering effective palliative care to older people with dementia. Journal of Clinical Nursing 17:9, 1144-1163. [CrossRef]

2. Gemma MM Jones, William J van der Eerden. 2008. Designing care environments for persons with Alzheimer's disease: visuoperceptual considerations. Reviews in Clinical Gerontology 18:01, 13. [CrossRef]

3. Sophie Pautex, François R. Herrmann, Paulette Le Lous, Monia Ghedira, Gilbert B. Zulian, Agnès Michon, Gabriel Gold. 2007. SYMPTOM RELIEF IN THE LAST WEEK OF LIFE: IS DEMENTIA ALWAYS A LIMITING FACTOR?. Journal of the American Geriatrics Society 55:8, 1316-1317. [CrossRef]

4. Eva Jakobsson , Ingrid Bergh , Fannie Gaston-Johansson, Carl-Magnus Stolt , Joakim Öhlén . 2006. The Turning Point: Clinical Identification of Dying and Reorientation of CareThe Turning Point: Clinical Identification of Dying and Reorientation of Care. Journal of Palliative Medicine 9:6, 1348-1358. [Abstract] [PDF] [PDF Plus]

5. Jenny T. van der Steen, Ladislav Volicer, Debby L. Gerritsen, Robin L. Kruse, Miel W. Ribbe, David R. Mehr. 2006. Defining severe dementia with the Minimum Data Set. International Journal of Geriatric Psychiatry 21:11, 1099-1106. [CrossRef]

6. Sharon E. Engel, Dan K. Kiely, Susan L. Mitchell. 2006. Satisfaction with End-of-Life Care for Nursing Home Residents with Advanced Dementia. Journal of the American Geriatrics Society 54:10, 1567-1572. [CrossRef]

7. S. Pleschberger. 2006. Palliative Care in Pflegeheimen—. Zeitschrift für Gerontologie und Geriatrie 39:5, 376-381. [CrossRef]

8. Susan L. Mitchell, Dan K. Kiely, Richard N. Jones, Holly Prigerson, Ladislav Volicer, Joan M. Teno. 2006. Advanced Dementia Research in the Nursing Home: The CASCADE Study. Alzheimer Disease \& Associated Disorders 20:3, 166-175. [CrossRef]

9. J. Brandon Wallace, Suzanne S. Prevost. 2006. Two Methods for Predicting Limited Life Expectancy in Nursing Homes. Journal of Nursing Scholarship 38:2, 148-153. [CrossRef]

10. Jennifer L. Lamberg, Carmel J. Person, Dan K. Kiely, Susan L. Mitchell. 2005. Decisions to Hospitalize Nursing Home Residents Dying with Advanced Dementia. Journal of the American Geriatrics Society 53:8, 1396-1401. [CrossRef] 